\title{
Les suites Isogènes (Isogenic sequences)
}

\author{
PAR M. MALLET \\ Inglineur des Ponts et Chausstes \\ English synopsis p. 5
}

M. Mallet, Ingénieur en Chef du Service Central des Etudes Générales et Grands Travaux, au Service de la Colonisation et de l'Hydraulique en Algérie, va faire paraître dans les éditions de "La Houille Blanche " un livre de mathématiques qui rassemblera et étudiera les fonctions auxquelles peut avoir affaire un Hydraulicien ou un Constructetr désirant tirer le maximum de la théorie. Des premiers chapitres relatifs aux fonctions de BERNOUILLI et d'EULER, nous extrayons un chapitre de synthèse qui montre les raisons de l'intérêt de telles fonctions et que nous sommes heureux de présenter à nos lecteurs.

M. MALLET étudie dans cet extrait les suites "Isogènes ". Une suite de fonctions est dite isogène lorsque chacun de ses termes est la dérivée du suivant. Si l'on impose à une telle suite une condition supplémentaire, par exemple de satisfaire à une équation aux différences finies out aux sommes finies, on trouve comme seule solution les suites de BerNovilli ou d'Eui.er. Ces équations aux différences finies interviennent dans certains problèmes de physique afpliquée et ont été utilisées assez récemment en Afrique du Nord pour le calcul des infiltrations sous le barrage du Sarno et la répartition des efforts dans la poutre précontrainte du pont de Djedeida en Tunisie.

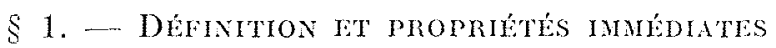

Nous appellerons suite isogene une suite indéfinie de fonctions :

$$
f_{0}(x) ; f_{1}(x) ; f_{2}(x) ; \ldots f_{n}(x) ; \ldots
$$

telle que :

$$
f_{n}^{\prime}(x)=f_{n-1}(x)
$$

la notation $f^{\prime}$ désignant la dérivée première par rapport à $x$ de la fonction $f$.

Le terme général de la suite isogène la plus simple est :

$$
f_{n}(x)=\frac{(x+h)^{n}}{n !}
$$

Une suite isogène polynomiale est donc de la forme :

$$
f_{n}(x)=\stackrel{n}{\Sigma}_{s=0}^{n}(n-s) ! x^{n-s}
$$

étant convenu, suivant l'usage, que $o !=1$.

Soient $\mathrm{P}_{n}(x)$ et $\mathrm{Q}_{n}(x)$ les nieme polynómes entiers (1) de deux suites isogènes (P) et $(Q)$ :

(1) Nous ne considérons que des polynômes entiers, nous nous dispenserons souvent de mettre le qualificatif entier.

$$
\begin{aligned}
& \mathrm{P}_{n}(x)=\mathrm{\Sigma} \frac{p_{\lambda} x^{x^{n-\lambda}}}{(n-\lambda) !} \\
& \mathrm{Q}_{n}(x)=\mathrm{\Sigma} \frac{q_{\mu} x^{n-\mu}}{(n-\mu) !}
\end{aligned}
$$

Considérons la somme :

$$
\mathrm{F}(n)=\Sigma(-1)^{s} \mathrm{P}_{s}(x) \mathrm{Q}_{n-s}(x)
$$

Je dis que cette somme est une constante; en effet, nous constatons de suite que la dérivée par rapport à $x$ est nulle.

Envisageons maintenant la somme :

$$
\mathrm{R}_{n}\left(x^{2}\right)=2^{n} \mathrm{\Sigma} \mathrm{P}_{s}(x) \mathrm{Q}_{n-s}(x)
$$

Je dis que $\mathrm{R}_{n}(x)$ est le terme général d'une nouvelle suite isogène polynomiale. En prenant les dérivées, nous vérifierons que :

$$
\mathrm{R}_{n}{ }^{\prime}(x)=\mathrm{R}_{n-1}(x)
$$

Revenons alors à $\mathrm{P}_{n}(x)$, on peut écrire : (3) $\mathrm{P}_{n}(x)=x_{0} \mathrm{Q}_{n}(x)+\alpha_{1} \mathrm{Q}_{n-3}(x)+\ldots+\alpha_{n} \mathrm{Q}_{0}(x$,

La détermination des coefficients $\alpha_{0}, \alpha_{1}, \ldots \alpha_{3}$ résulte de l'identification des diverses puissances en $x$ en appliquant la relation (3) aux diverses valeurs $n=0,1,2 \ldots n$. 
(4)

$$
\left\{\begin{array}{l}
p_{0}=\alpha_{0} q_{0} \\
p_{1}=\alpha_{1} q_{0}+\alpha_{0} q_{1} \\
\ldots \ldots \ldots \ldots \\
p_{s}=\Sigma \alpha_{i} q_{s-i} \\
\ldots \ldots \ldots \ldots
\end{array}\right.
$$

Réciproquement nous pourrons écrire :

$(5) \mathrm{Q}_{n}(x)=\beta_{0} \mathrm{P}_{n}(x)+\beta_{1} \mathrm{P}_{n-1}(x)+\ldots+\beta_{n} \mathrm{P}_{0}(x)$

et nous aurons :

$$
\left\{\begin{array}{l}
q_{0}=\beta_{0} p_{0} \\
q_{1}=\beta_{1} p_{0}+\beta_{0} p_{1} \\
\ldots \ldots \ldots \ldots \\
q_{s}=\Sigma \beta_{i} p_{s-i}
\end{array}\right.
$$

La liaison entre les $\alpha$ et les $\beta$ se détermine immédiatement, c'est :

$$
\left\{\begin{array}{l}
\alpha_{0} \beta_{0}=1 \\
x_{1} \beta_{0}+\alpha_{0} \beta_{1}=0 \\
\ldots \ldots \ldots \ldots \\
\Sigma \alpha_{i} \beta_{s-i}=0 \\
\ldots \ldots \ldots \ldots
\end{array}\right.
$$

Ainsi donc $p_{0}$ et $q_{0}$ étant deux nombres non nuls (par définition des suites isogènes), on en déduit que les $\alpha$ et $\beta$ sont bien déterminés. Les équations (7) montrent en outre que si les $\alpha$ sont choisis arbitrairement (sous la réserve que $\alpha_{0}$ ne peut être pris égal à zéro), les $\beta$ seront bien déterminés.

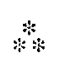

\section{\$2. - Suites isogìnes particulières}

Supposons que la suite isogène $\mathrm{P}_{n}(x)$ soit assujettie à vérifier l'équation fonctionnelle :

$$
P_{n}(x+1)-P_{n}(x)=\Omega_{n-2}(x)
$$

les polynômes $\Omega_{n}(x)$ formant eux aussi une suite isogene. Il est facile de voir que ce probleme a une solution et une seule; en effet, nous déduisons de suite de (8), grâce à la formule de Taylor :

$$
\begin{gathered}
\Omega_{n-1}(x)=\frac{1}{1 !} \mathrm{P}_{n-1}(x)+\frac{1}{2 !} \mathrm{P}_{n-2}(x)+\ldots \\
+\frac{1}{n !} \mathrm{P}_{0}(x)
\end{gathered}
$$

Autrement dit, nous connaissons le développement des polynomes $\Omega(x)$ en fonction des polynòmes $\mathrm{P}(\boldsymbol{x})$; par suite, nous avons :

(9) $\mathrm{P}_{n}(x)=\alpha_{0} \Omega_{n}(x)+\alpha_{1} \Omega_{n-1}(x)+\ldots+\alpha_{n} \Omega_{0}(x)$

et les a se calculent à partir des équations (7), sachant que :

$$
\beta_{0}=\frac{1}{1 !} ; \beta_{1}=\frac{1}{2 !} ; \beta_{2}=\frac{1}{3 !} ; \ldots
$$

Nous ferons d'aboid la remarque que les $\alpha$ et les $\beta$ sont indépendants des coefficients de $\Omega$, et nous écrirons à nouveau les équations (7) en donnant aux $\beta$ leur valeur réelle :

$(10)\left\{\begin{array}{l}\alpha_{0} \cdot \frac{1}{1 !}=1 \\ \ldots \ldots \ldots \ldots \ldots \ldots \ldots \\ \alpha_{0} \cdot \frac{1}{2 !}+\alpha_{1} \frac{1}{1 !}=0 \\ \alpha_{0} \cdot \frac{1}{(s+1) !}+\alpha_{1} \frac{1}{s !}+\alpha_{2} \frac{1}{(s-) 1 !}+\ldots \ldots=0 \\ \ldots \ldots \ldots \ldots \ldots \ldots \ldots \ldots \ldots \ldots \ldots \ldots \ldots \ldots \ldots\end{array}\right.$

Si nous posons :

$\alpha_{0}=1 ; \quad \alpha_{1}=\frac{\gamma_{1}}{1 !} ; \quad \alpha_{2}=\frac{\gamma_{2}}{2 !} ; \ldots \alpha_{s}=\frac{\gamma_{s}}{s !} ; \ldots$

l'équation générale du système (10) devient :

$1+\frac{s+1}{1 !} \gamma_{1}+\frac{(s+1) s}{2 !} \gamma_{2}+\ldots+\frac{s+1}{1} \gamma_{s}=0$

ou encore en notation symbolique :

$$
((\gamma+1))^{s: 1}-\gamma_{s: 1}=0 .
$$

Les nombres $\gamma$ ainsi déterminés sont identiques aux nombres de Bernouilli car les équations de recurrence (10) admettent un ensemble unique de solutions.

Ainsi, et il convient d'insister un peu, quelque soit la suite isogène $\Omega_{n}(x)$, la suite isogène $\mathrm{P}_{n}(x)$, solution de l'équation fonctionnelle (8) est donnée par :

$\mathrm{P}_{n}(x)=\Omega_{n}(x)+\frac{\mathcal{B}_{1}}{1 !} \Omega_{n-1}(x)+\frac{\mathcal{B}_{2}}{2 !} \Omega_{n-2}(x)+\ldots$

Or, nous avons vu que $((\boldsymbol{B}+z))^{n}$ est le seul polynome (a une constante près) satisfaisant a :

$$
((\mathcal{B}+z+1))^{n}-((\mathcal{B}+z))^{n}=n z^{n-1}
$$

par suite l'équation fonctionnelle :

$$
\mathrm{P}_{n}(x+1)-\mathrm{P}_{n}(x)=\frac{x^{n-1}}{(n-1) !}
$$


armet comme unique solution polynomiale la suite isogène de Bernouilli :

$$
B_{n}(x)=\frac{((B+x))^{n}}{n !}
$$

Ia suile isogine, solution unique de :

$$
\begin{gathered}
P_{n}(x+1)-P_{n}(x)=\Omega_{n-1}(x)=(1) \frac{x^{n-1}}{(n-1) !} \\
+(1)_{1} \frac{x^{n-2}}{(n-2) !}+{ }^{(1)} \frac{x^{n-3}}{(n-3) !}+\cdots
\end{gathered}
$$

est done, dapres (11) et (12,13) foumie par :

$$
P_{n}(x)=2 \frac{B S Q_{n-s}(x)}{s !}=2(1) B B_{n \rightarrow s}(x)
$$

Si nous prenons pour suite isogine $\Omega$ la suite de Bernouilli, nous aurons :

$$
\begin{aligned}
B_{n-1}(x) & =\frac{x^{n-1}}{(n-1) !}+\frac{\left(B_{1} x^{n+2}\right.}{1 !(n-2) !} \\
& +\frac{\left(B_{3} x^{n-3}\right.}{2 !(n-3) !}+\ldots
\end{aligned}
$$

ce qui conduil, d'après (14), à la solution :

$$
P_{n}(x)=\Sigma \frac{\mathcal{B}_{s} B_{n-s}(x)}{s !}
$$

or, il est facile de trouver directement runique solution en prenant pour $\mathrm{P}_{n}(x)$ un polynòme du degré $n$ formé à parlir de $B_{n-1}(x)$ et de $B n(x)$ :

$$
P_{n}(x)=(x-1) B_{n-1}(x)-(n-1) B_{n}(x) .
$$

On a done:

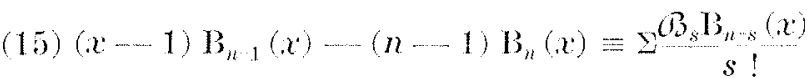

Nous allons génémaliser celte remarquable identilé.

Considerons à cet effet l'équation :

$\mathrm{P}_{n}(x+h+1)-\mathrm{P}_{n}(x+h)=\Omega_{n-1}(x+h)$.

Le deuxieme membre peut s'écrire en développant en série de laylor :

$$
\begin{gathered}
\Omega_{n-1}(h)+\frac{x}{1 !} \Omega_{n: 2}(h)+\frac{x^{2}}{2 !} \Omega_{n-3}(h)+\ldots \\
+\frac{x^{n-1}}{(n-1) !} \Omega_{n}(h)=\Omega_{n-1}(x)+\frac{h}{1 !} \Omega_{n-2}(x) \\
+\frac{h^{2}}{2 !} \Omega_{n-3}(x)+\ldots
\end{gathered}
$$

Nous aurons done, d'après (12) et (14)

$(16) \mathrm{P}_{n}(x+h)=\Sigma \Omega_{n-s}(h) \cdot \mathrm{B}_{s}(x)=-2 \mathrm{~B}_{x}(h) \Omega_{n-s}(x)$ or, nous savoms que pour :

$$
\mathrm{Q}_{n}(x)=\mathrm{B}_{i}(x)
$$

(1) 1 :

$P_{n}(x+h)=(x+h-1) B_{n-1}(x+h)$

$$
-(n-1) \mathrm{B}_{n}(x+h) \text {. }
$$

Posant $h=y$, il vient :

$$
\begin{gathered}
(17)(x+y-1) \mathrm{B}_{n-1}(x+y) \cdots(n-1) \mathrm{B}_{n}(x+y) \\
\equiv \Sigma \mathrm{B}_{n-s}(x) \mathrm{B}_{s}(y)
\end{gathered}
$$

Cette identite, que l'on aurait pu obtenir par application de la formule (19, I), fommira un srand nombre de relations entre les $B$, en parliculier pour $x=y=0$ et $x=y=1 / 2$.

Le premier cas conduira à :

$$
-n \mathcal{S}_{n-1}-(n-1) \mathfrak{B}_{n}=2 n_{s} \mathfrak{B}_{s} \mathcal{B}_{n-s}
$$

et le second à :

$$
-2^{n}-n\left(B_{s-1}= \pm n_{s}\left(2^{*}-1\right) \mathfrak{B}_{s} \boldsymbol{B}_{n-s}\right.
$$

Nous retrouverons plus loin ectle égalité.

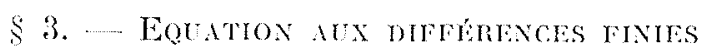

Posons :

$$
D /(x)=f(x+1)-f(x)
$$

notls aturons :

$$
\begin{aligned}
D * f(x) & =\mathrm{D} /(x+1)-\mathrm{D} /(x) \\
& =\int(x+2)-2 /(x+1)+f(x)
\end{aligned}
$$

Nous allons montrer (que :

$$
\begin{gathered}
\mathrm{D}^{n} f(x)=f(x+n)-n_{1} /(x+n-1) \\
-1+n(x+n-2)-\ldots
\end{gathered}
$$

En effet, faisons pour un instant :

$$
\begin{gathered}
D^{n} f(x)=f(x+n)-c_{1} f(x+n-1) \\
+c_{2} /(x+n-2)-\ldots
\end{gathered}
$$

nous vérifierons par récurence que les coefficients $C$ ne dépendent pas de la fonction $f(x)$, prenant alors pour les calculer :

$$
f(x)=e^{x}
$$

nous obtiendrons de suite :

$$
\begin{aligned}
& \mathrm{D} e^{*}=e^{x}(e-1) \\
& \mathrm{D}^{*} e^{\prime \prime}=e^{*}(e-1)^{\prime} \\
& \ldots \ldots \\
& \mathrm{D}^{\prime \prime} e^{\prime}=e^{*}(e-1)^{\prime \prime}
\end{aligned}
$$

ce qui nous conduit bien aux coefficionls du binôme. 


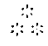

Au baragraphe 2, nous arons résolu l'ćpuatlion:

$$
D /(x)=(n-1) !
$$

lorsquon se borne a lat recherche des solutions polynomiales. Nous verrons plus tard comment resondre le problime général.

Nous arons louré

$$
\left.f_{1}(x)=13, x\right)
$$

Petis nous aroms eherehe dans les momes conditions lat solution de:

$$
\text { D) } P(x)=B_{n}(x)
$$

donc, en latl, la solulion de lépualion du denxiome ordre:

$$
1)^{2} f(x)=\left(\begin{array}{c}
x^{n}-2 \\
(n-2) !
\end{array}\right.
$$

re qui nous a donné :

$$
\begin{gathered}
f_{2}(x)=(x-1) B_{n-1}(x) \cdots(n-1) B_{H}(x) \\
= \pm \quad B_{s} B_{n, x}(x)
\end{gathered}
$$

Nous appollerons $B_{n}=(x)$ le polynome $f: x(x)$ de degre $n$, les résulials prédents mots permellont dierire:

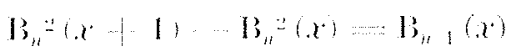

et mous avoms momlar que:

$$
\begin{aligned}
& \text { (21) } \quad B_{n}-(x+y)= \pm B_{s}(x) B_{n s}(y) \\
& =(x+y-1) B_{n-1}(x+y)-(n-1) B_{n}(x+y)
\end{aligned}
$$

Si nous cherchons a resoudre l'apuation du troisiome ordre:

$$
G_{i j}(x+1) \quad G_{i j}(x)=13^{2}{ }^{2}(x)
$$

mous serons eonduit à une égalití analogue a (21). Nous appellerons $B_{3} "(x)$ le $n^{\text {iam }}$ polynome de la suile isogiene, solution de celle equalion; nots atuons :

(2:) $\mathrm{B}_{3} 3(x+y+z)= \pm \mathrm{B}_{p}(x) \mathrm{B}_{i}(y) \mathrm{B}_{i}(z)$

arec la seule nécessité pour les entiers posilifs ou nuls $p, q, r$ de salisfaire:

$$
p+q+r=n,
$$

la somme z s'elendant à loules es valeurs possibles de $p, q, r$.

Nous calculerons directement $B_{n}:(x)$ comme nots l'avions fait pour $B_{n}{ }^{2}(x)$ et nous trouverons :
(2)

$$
\begin{aligned}
& \left.B_{n}: x(x)=(x-1), x-1, b_{n} \cdot x\right) \\
& -(n-2)(x-3) B_{n-1}(x) \\
& +\frac{1}{2}(n-1)(n-2) B_{n}(x) .
\end{aligned}
$$

Mais nous aturons atussi, dapres (29):

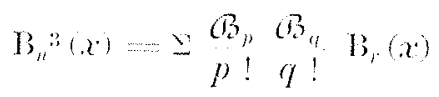

la somme sétendant à toutes les valeurs ontieres posilives ou nulles de $p, q, r$, salishaisant a :

$$
\text { l) }+q+r=n
$$

Cost ansi que lon anra:

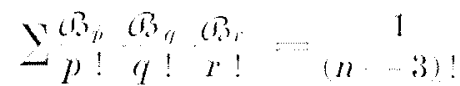

$$
\begin{aligned}
& \left|\begin{array}{cc}
1 & B_{n} \\
2 n & +3 B_{n-1} \\
2 n n-1
\end{array}+\frac{B_{n 2}}{n-2}\right|
\end{aligned}
$$

Appelons $B_{\|}{ }^{\prime}(x)$ le polynòme qui satisfait à :

$$
\mathrm{B}_{n}^{4}(x+1) \cdots \mathrm{B}_{n}^{4}(x)=\mathrm{B}^{3}+\mathrm{I}(x)
$$

Un calcul analogue aux préedents dome alors:

$$
B_{n}^{\prime}(x)= \pm \frac{B_{n} B_{n} B_{r}}{p ! q ! \gamma !} B_{x}(x)
$$

ave lobligation, pour les nombres enliers posilili's ou nuls $p, q, r, s$, de sollisfaire :

$$
\text { p) }+q+r+s=n \text {. }
$$

Nous savons done former une solulion polynomiale de licqualion:

$$
1) \int(x)=\frac{x^{2 n} 1}{(n-1) !}
$$

Cette équation aux diférences finies du quatrieme ordre joue un rôle extrimencnt imporlant en physique appliquée. C'est pourquoi nous nous sommes étendus peut-être un peu lrop longuement sur la thérie des polynomes d'Euler ct de Bernouilli.

$$
\therefore
$$

\section{Eomtroxs nUX somms rantes}

Considerons maintenant une suite isogene assujellic à rérifier :

(26) $\quad V_{n}(x+1)+V_{n}(x)=\Omega_{n}(x)$ 


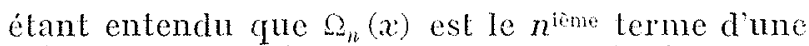
suite également isogene. Nous en déduisons :

$$
\begin{gathered}
g_{n}(x)=2 V_{n}(x)+\frac{1}{1 !} V_{n-1}(x) \\
+\frac{1}{2 !} V_{n 2}(x)+\ldots
\end{gathered}
$$

autrement dit on connait le développement de $\Omega_{n}(x)$ en fonction de $\mathrm{V}_{p}(x)$ :

$\Omega_{n}(x)=\beta_{0} V_{n}(x)+\beta_{1} V_{n-1}(x)+\beta_{2} V_{n}(x)+\ldots$ ayee:

$$
\beta_{11}=2 ; \beta_{1}=\frac{1}{1 !} ; \beta_{2}=\frac{1}{2 !} ; \ldots
$$

Nous savons que nous pourons alors cerire: $V_{n}(x)=\alpha_{0} Q_{n}(x)+\alpha_{1} Q_{n}(x)+\alpha_{n} \Omega_{n}(x)+\ldots$ el que les a se deduisant des $\beta$ par :

$$
\left\{\begin{array}{c}
\alpha_{0} \beta_{0}=1 \\
\ldots \ldots \\
\Sigma_{2 \beta} \beta_{s}=0
\end{array}\right.
$$

Nous avons done :

$$
s_{010}=\frac{1}{2}
$$

puis pour retation gincrale:

$$
\begin{gathered}
0=x_{11} \frac{1}{s !}+\mu_{1} \frac{1}{(s-1) !+\ldots} \\
+\alpha_{k-1} \frac{1}{1 !}+2 \mu_{*}
\end{gathered}
$$

en posant :

$$
\alpha_{i}=\frac{1}{2} \frac{\%}{i !}
$$

nous transformons la dite équation en :

$$
\begin{gathered}
\left.0 ! s !+1 !(s-1) !+\cdots+\frac{\gamma_{1}}{\gamma_{0}}+1 / 1\right) ! 1 ! \\
+\frac{2 \gamma_{s}}{s !}=0
\end{gathered}
$$

ou encore symboliquement :

$$
((\gamma+1))^{*}+\gamma^{s}=0 \text {. }
$$

Sachant que $\gamma_{0}=1$, cette relation $(27)$ définit les nombres $\approx$ que nous avons ćludiés précédemment au chapilie II.

Nous formons done ainsi le polynome $V_{n}(x)$ :

$$
\begin{aligned}
V_{n}(x) & =\frac{1}{2} \mid \Omega_{n}(x)+\frac{\vartheta_{1}}{1 !} \Omega_{n 1}(x) \\
& +\frac{\widetilde{\vartheta}_{3}}{3 !} \Omega_{n 3}(x)+\ldots \mid
\end{aligned}
$$

Au chapitre II, nous avons été conduits à la relation :

$$
((x+x+1))^{n}+((8+x))^{n}=2 x^{n}
$$

par suite nous pouvons affirmer que :

$$
{ }^{r} \mathrm{I}_{n}(x)=\frac{1}{n !}((⿻+x))^{n}
$$

définit la seule suite polynomiale isogene satisfaisant à l'équation fonctionnelle :

$$
f(x+1)+f(x)=2 \frac{x^{n}}{n !} .
$$

Nous dirons, pour simplifier l'exposé, que Th est la suite isogine druler.

$$
\because
$$

La solution de l'équation fonctionnelle:

$$
\begin{aligned}
& V_{n}(x+1)+V_{n}(x)=2 \Omega_{n}(x) \\
= & 2\left[\omega_{0} \frac{x^{n}}{n !}+\omega_{1} \frac{x^{n-1}}{(n-1) !}+\cdots\right]
\end{aligned}
$$

sera done fournie par l'une on l'autre des sommes :

(32) $V_{n}(x)= \pm \frac{\ddot{o s}_{s}}{s !} Q_{n-s}(x)=\Sigma(0) T_{n-k}(x)$.

Cela blant, nous essayerons de former comme précedemment la suite isogine, solution de l'equation:

(33) $\quad V_{n}(x+1)+V_{n}(x)={ }^{\top} \mathrm{T}_{n}(x)$

solution que nous appellerons $\mathrm{T}_{n}=(x)$.

Nous trouverons, d'une part, par recherehe directe :

(84) $\mathrm{T}{ }^{2}{ }^{2}(x)=(n+1) \mathrm{T}_{n+1}(x)-(x-1) \mathrm{T}_{n}{ }^{\prime}(x)$

et d'atutre part, par applieation de la formule (29) :

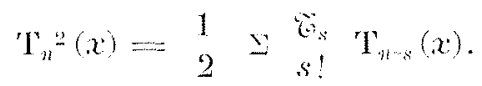

L'egalité des seconds membres des formules (34) et (35) conduira à un cerlain nombre de relations fort intéressantes; en particulicr pour $x=0$, on aura :

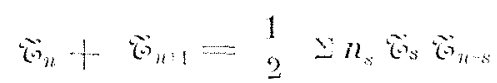

$$
\text { : }
$$

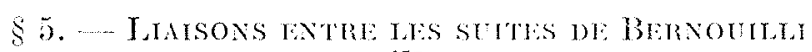
I:T D'Lutia

Nous avoms vu que la suite de Bernomilli salisfail à :

$$
\mathrm{D} /(x)=\frac{x^{n-1}}{(n-1) !}
$$


el que celle d'Euler founil les solutions polynomiales de :

$$
\mathrm{S} /(x)=2 \frac{x^{n}}{n !}
$$

Lidéce la plus immédiale el qui peut être generalisce conduit a rechercher des solutions de :

$$
\operatorname{SD} f(x)=2 \frac{x^{n-1}}{(n-1) !}
$$

cest-it-dire dans le champ limite qui nous interesse à trourer les solutions génératrices d'une suile isogene de polynomes de l'une ou l'autre des équations :

$$
D /(x)=T_{n-1}(x)
$$

$$
S g(x)=2 B_{11-1}(x)
$$

Or, la solution de (385) est facile à trouver, l'équation s'écrit en effet :

$$
f(x+2)-f(x)=2 \frac{x^{n-1}}{(n-1) !}
$$

ou encore arec $x=2 y$ :

$$
f(2 y+1)-f(2 y)=2^{n} \frac{y^{n-1}}{(n-1) !}
$$

la solution polynomiale est par suile :

$$
\left\{\begin{array}{l}
f(2 y)=2 n B_{n}(y) \\
f(x)=2{ }^{n} B_{n} \quad x \\
2
\end{array}\right.
$$

Nous aurons done tout d'abord, d'apres (39) ct $(40)$ :

$$
\begin{aligned}
& \mathrm{T}_{n-1}(x) \equiv 2^{n}\left[\mathrm{~B}_{n}\left(\frac{x+1}{2}\right)-\mathrm{B}_{n}\left(\frac{x}{2}\right)\right] \\
& 2 \mathrm{~B}_{n}(x) \equiv 2^{n}\left[\mathrm{~B}_{n}\left(\frac{(x+1}{2}\right)+\mathrm{B}_{n}\left(\begin{array}{l}
x \\
2
\end{array}\right)\right]
\end{aligned}
$$

Puis, en application de la relation (16):

$$
\begin{aligned}
2^{n} \mathrm{~B}_{n}\left(\frac{x+y}{2}\right) & \equiv \pm \mathrm{T}_{n, s}(x) \mathrm{B}_{s}(y) \\
& \equiv \Sigma \mathrm{T}_{n * s}(y) \mathrm{B}_{*}(x) .
\end{aligned}
$$

Les relations (42) et (43) sont assez curieuses, nous retrouverons (42) par la suile.

Quant à l'équation (41), elle n'est qu'un cas particulier d'une formule due a Raabe.

Cette formule de Rabbe dome la valeur de la somme :

$$
(2 p) n+\sum_{s=0}^{2 n-1}(-1-1) s \mathrm{~B}_{n}\left(\begin{array}{c}
x+s \\
2 p
\end{array}\right)
$$

Ia dimonstration en est facile, appelons $\mathrm{E}(\boldsymbol{x})$ l'expression ci-dessus et formons :

$$
\begin{aligned}
& \left(\quad B_{n}\left(\begin{array}{c}
x+1 \\
2 p
\end{array}\right)-B_{n}\left(\frac{x+2}{2 p}\right)+B_{n}\left(\frac{x+3}{2 p}\right) \cdots \ldots\right. \\
& (2 p) n \mathrm{E}(x+1)+\mathrm{E}(x)]=\left\{\mathrm{B}_{n}\left(\begin{array}{c}
x \\
2
\end{array}\right) \mathrm{B}_{n}\left(\begin{array}{c}
x+1 \\
2 p
\end{array}\right)+\mathrm{B}_{n}\left(\begin{array}{c}
x+2 \\
2 \eta
\end{array}\right) \cdots \mathrm{B}_{n}\left(\begin{array}{c}
(x+3 \\
2 p
\end{array}\right)+\ldots\right. \\
& =B_{n}\left(\begin{array}{c}
x \\
2 p
\end{array}\right)-\mathrm{B}_{n}\left(\frac{x+2 p}{2 p}\right)=\frac{x^{n-1}}{(2 p)^{n-1}(n-\cdots-1) !}
\end{aligned}
$$

Par suite:

$$
\mathrm{E}(x+1)+\mathrm{E}(x)=\frac{x^{n-1}}{(n-1)}
$$

La valeur de lexpression $\mathrm{F}(x)$ est done connue, c'est :

$$
\mathrm{I}(x)=\frac{1}{2} \mathrm{~T}_{n-1}(x)
$$

il en résulte que nous avons :

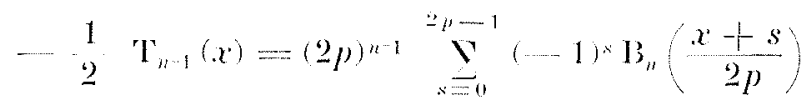

Cate expression, plus générale que (41), est connue sous to nom de Iroisieme formule de Raabe.

\section{Exercices du Chapilie $\mathrm{IV}^{\mathrm{r}}$}

$1^{\circ}$ Il existe un grand nombre de suites isogènes polynomiales présentant un grand intérèt en Physique appliquée. Nous aurons l'occasion d'en étudier ultérieurement un certain nombre. En particulier nous nous arrêterons sur les poIynomes d'Hermite que nous définirons par la relation :

$$
\frac{d^{n}}{d x^{n}} e^{-x^{2}}=(-1)^{n} n ! 2^{n} \mathrm{H}_{n}(x) e^{-x^{n}}
$$

Ces polynomes $H_{n}(x)$ forment une suite isogine qui satisfait à un cerlain nombre de relalions remarquables.

(a) Démontrez que :

$$
\int_{-\infty}^{+\infty} \mathrm{H}_{y}(x) \mathrm{H}_{1}(x) \mathrm{H}_{1}(x) e, d x=0
$$


si les entiers $p, q$, $r$ chant rangés par ordre croissant, le nombre $\frac{1}{2}(p+q-r)$ n'est pas un entier posilil ou nul.

b) $\mathrm{Si} \stackrel{1}{2}(p+q-r)$ est un contior positil on nul, on peul poser :

$$
\begin{aligned}
& a=\frac{1}{2}(p+q-r) \\
& b=\frac{1}{2}(p-\cdots+r) \\
& c=\frac{1}{2}(p+q+r)
\end{aligned}
$$

$a, b, c$ ctant des entiers positifs ou nuls, montre\% que:

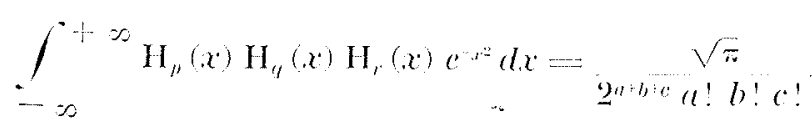

(c) Demontrez la relation:

$$
2: \mathrm{H}_{x}(\sqrt{2}, x)=\sum_{n=0}^{n=s} \mathrm{H}_{n}(x) \mathrm{H}_{s-n}(x)
$$

$$
\because
$$

$2^{\circ}$ En posant symboliquement :

$$
(\mathrm{C}+1))^{n+1}-\mathrm{C}_{n}=\frac{1}{2}
$$

montrer que les nombres $C_{n}$ ainsi dólinis sont lics aux nombres ö par :

$$
\begin{aligned}
& C_{n}=\frac{1}{2} \ddot{n}_{n} \\
& \because \cdots
\end{aligned}
$$

3" $h(z)$ clant un polynome entier en z, montree (floe :

$$
h(z)=\mathrm{H}(1)-\mathrm{H}(0)+2 \lambda_{i} \mathrm{~B}_{i}(z)
$$

en posint :

$$
\mathrm{H}(x)=\int h(x) d x
$$

4" $n$ chant le nombre des variables indipendanles $x, y, z \ldots$;

$\varepsilon_{i}$ des quantilés cigales a $=1$, soit $S_{p}$ la somme des 2" fonclions de la forme :

$$
\varepsilon_{1} \varepsilon_{2} \ldots \varepsilon_{n}\left(\varepsilon_{1} x+z_{n} y+\varepsilon_{n} z+\ldots\right)_{n} .
$$

On alla: :

$$
S_{p}=0
$$

si $p$ est un entier non nul inlérieur it $n$.

si $p$ est un colier supéricur oul bisil a $n$, on alura:

$$
\begin{aligned}
& S_{n}=2 " n ! x y z \\
& S_{1,2, y ! 1}=0 \\
& S_{n=2}=2 \begin{array}{c}
(n+2) ! \\
3 !
\end{array} x z \ldots(x=-1, y=+z+\ldots)
\end{aligned}
$$

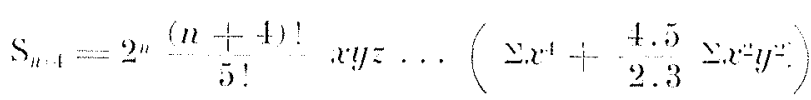

5" Soit l'équalion:

$$
x^{*}-1=0 .
$$

Appelons; ane racine primilive el considerons les 2 sommes de la forme :

$$
\sigma=1 \pm ?+q^{2} \ldots \ldots \pm ?^{* 1}
$$

Nolons que:

$$
1-\frac{1}{2}=
$$

est une sommo de la mêne famille. Considérons alors la suite isogine de polynomes :

$$
P_{n}(x)=\frac{1}{n !} \geq\left(x-\frac{5}{2}\right)^{n}
$$

montrer en appliquant soit la formule symbolique fondamentale, soit la theorie des suites isogènes, soit les résultats du chapitre 2 (paragraphe 6$)$, que :

$$
\begin{aligned}
\mathrm{P}_{n}(x) & =2 \times \mathrm{T}_{n}(x)+\frac{1}{2 !}=\left(\begin{array}{l}
\sigma \\
2
\end{array}\right)^{\prime \prime} \mathrm{T}_{n-2}(x) \\
& +\frac{1}{4 !} \geq\left(\frac{\sigma}{2}\right)^{4} \mathrm{~T}_{n+4}(x)+\ldots
\end{aligned}
$$

$$
\because
$$

6" On est conduit par lexercice 5 a lexaluation de :

$$
\because \sigma^{2} m
$$

montrer que ces sommes sont nulles si m nost pas un mulliple des.

7" Montrer que s elant impair :

$$
\dddot{*}=2 \pm 1 \leq \sigma^{*} \text {. }
$$


so Potr: $s=3$, on a :

$$
\begin{aligned}
& y\left(\frac{\sigma}{2}\right)^{2}=0 ; \quad y\left(\begin{array}{c}
\sigma \\
2
\end{array}\right)^{m \cdot 2}=0 \\
& د\left(\begin{array}{c}
5 \\
2
\end{array}\right)^{1}=0 ; \quad y\left(\begin{array}{c}
5 \\
2
\end{array}\right)^{m s k}=0 \\
& y\left(\begin{array}{l}
\sigma \\
2
\end{array}{ }^{3}=3 ; \quad y\left(\begin{array}{c}
\sigma \\
2
\end{array}\right)^{m \text { mis }}=3\right.
\end{aligned}
$$

le sorle que:

$$
\begin{aligned}
\frac{1}{3} \mathrm{P}_{n}(x)=\frac{1}{3} 2 \times 1 \mathrm{~T}_{n}(x) & +\frac{1}{6 !} \mathrm{T}_{n \cdot 6}(x) \\
& +1 \frac{1}{12 !} \mathrm{T}_{n+12}(x)+\ldots
\end{aligned}
$$

Expliciler celle relation pour $x=0$ et $x=\frac{1}{2}$

$9^{\circ}$ Considérons maintenant les $2-1$ sommes de la forme:

$$
\sigma=1 \pm \rho \pm \rho^{2} \pm \ldots \pm q^{s}
$$

et appelons a le nombre de signes négalifs que contient celle somme.

Délinissons par :

$$
(n+s+1) ! Q_{n}(x)=2(-1) x\left(x-\frac{5}{2}\right)^{n+1}
$$

une suite isogine de polynòmes.
Posons $m=n+s+1$. Nontrer que :

$$
\begin{aligned}
\frac{1}{2} Q_{n}^{\prime}(x)= & \frac{1}{1 !} \geq(-1) \times \frac{\sigma}{2} B_{m+1}(x) \\
& +\frac{1}{3 !} \cup(-1) *\left(\frac{\sigma}{2}\right)^{:} B_{m: 3}(x)+\ldots
\end{aligned}
$$

10" On est donc conduit à l'élude des somnles:

$$
\pm(-1) *\left(\frac{\sigma}{2}\right)^{2+1}
$$

Monlrer que ces sommes sont nulles si $(2 q+1)$ n'est pas un multiple de s.

11" Montrer que s elant imparir :

$(s+2)(s+3) \ldots 2 s \cdot n_{s+1}=2(-1) n\left(\begin{array}{l}5 \\ 2\end{array}\right)^{2 *}$

$12^{\circ}$ Pour $s=3$, on aura :

$$
m=n+4
$$

$$
\begin{aligned}
& \frac{1}{6} Q_{n}{ }^{\prime}(x)=\frac{1}{3 !} B_{n+1}(x)+\frac{1}{9 !} B_{n-3}(x) \\
& +\frac{1}{15 !} B_{n+11}(x)+\ldots
\end{aligned}
$$

Expliciter cette relation pour $x=0$ et $x=\frac{1}{2}$.

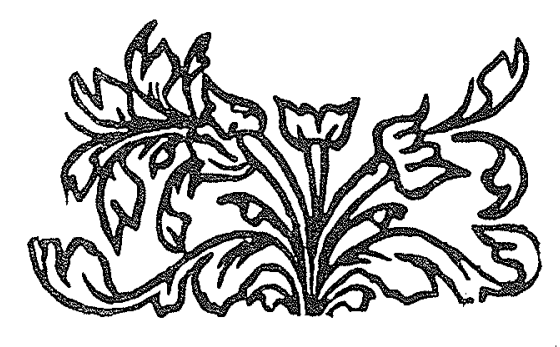

